\title{
Comparison of high-performance liquid chromatography and ultraviolet-visible spectrophotometry to determine the best method to assess Levofloxacin released from mesoporous silica microspheres/nano-hydroxyapatite composite scaffolds
}

\author{
QI WANG, GUODONG WANG, SHICHENG XIE, XIAOWEI ZHAO and YUANMIN ZHANG
}

Department of Orthopedics, The Affiliated Hospital of Jining Medical University, Jining, Shandong 272000, P.R. China

Received June 27, 2018; Accepted January 22, 2019

DOI: $10.3892 /$ etm.2019.7238

\begin{abstract}
An assessment of Levofloxacin by high-performance liquid chromatography (HPLC) or ultraviolet-visible spectrophotometry (UV-Vis) and its pharmacokinetics in serum or plasma was made in a previous study by the present authors. Levofloxacin-loaded mesoporous silica microspheres/nano-hydroxyapatite (n-HA) composite scaffolds comprise a novel synthetic composite scaffold that may be utilized as a drug-delivery system for clinical usage. However, few studies have been published concerning a comparison of HPLC with UV-Vis, which is the preferred method for determination of Levofloxacin. In the present study, an HPLC method was first established, and subsequently a comparison of HPLC with the UV-Vis method was performed. The standard curve was established, and recovery rate from simulated body fluid was calculated. The linear concentration range for Levofloxacin was $0.05-300 \mu \mathrm{g} / \mathrm{ml}$. The regression equation for HPLC was $y=0.033 x+0.010$, with $R^{2}=0.9991$, whereas that for UV-Vis was $y=0.065 x+0.017$, with $R^{2}=0.9999$. The recovery rates of low, medium and high $(5,25$ and $50 \mu \mathrm{g} / \mathrm{ml})$ concentrations of Levofloxacin determined by HPLC were $96.37 \pm 0.50$, $110.96 \pm 0.23$ and $104.79 \pm 0.06 \%$, respectively, whereas those for low, medium and high concentrations according to UV-Vis were $96.00 \pm 2.00,99.50 \pm 0.00$ and $98.67 \pm 0.06 \%$, respectively. Taken together, these findings demonstrated that it is not accurate to measure the concentration of drugs loaded on
\end{abstract}

Correspondence to: Professor Yuanmin Zhang, Department of Orthopedics, The Affiliated Hospital of Jining Medical University, 89 Guhuai Road, Jining, Shandong 272000, P.R. China

E-mail: zhangyuanmin11@126.com

Abbreviations: HPLC, high-performance liquid chromatography; UV-Vis, ultraviolet-visible spectrophotometry; MSN, magnetic mesoporous silica nanoparticle; n-HA, nano-hydroxyapatite; PU, polyurethane

Key words: Levofloxacin, mesoporous silica microspheres, composite scaffolds, high performance liquid chromatography, ultraviolet visible spectrophotometer the biodegradable composite composites by UV-Vis. HPLC is the preferred method to evaluate sustained release characteristics of Levofloxacin released from mesoporous silica microspheres/n-HA composite scaffolds. The present study also provides guidance on which methods should be selected for investigating the sustained release properties of drugs in tissue engineering. The accurate determination of drug concentration in the drug delivery system provides guidance for the treatment of infectious diseases.

\section{Introduction}

Levofloxacin belongs to the third generation of fluoroquinolone antibiotics (1), demonstrating typical broad-spectrum antibacterial properties. It possesses antibacterial properties against both Gram-positive and Gram-negative bacteria. The mechanism of action comprises inhibition of bacterial type II topoisomerase by interfering with the processes of DNA replication, transcription, repair and recombination (2). Therefore, it has been widely applied in the treatment of various systematic infections, including respiratory tract and urinary tract infections (3-5).

Levofloxacin has been selected as a component of the drug-delivery system in previous studies due to its low molecular mass (6). Magnetic mesoporous silica nanoparticles (MSNs) are nanostructured materials that have aroused widespread interest due to their potential application as a controlled delivery system for therapeutic molecules $(4,7,8)$. MSNs are characterized by having an adjustable surface, large pore volume, and controllable pore size. Different antibiotics and biologically active molecules, including DNA and RNA, may be loaded into MSNs easily (9). In a previous study by our research group, it was demonstrated that Levofloxacin could be successfully loaded into MSNs, and those MSNs were subsequently adsorbed onto the surface of nano-hydroxyapatite/polyurethane (n-HA/PU), which was then used as the drug-delivery system. A novel biodegradable, sustainable antibiotic release composites scaffold was synthesized (10).

High-performance liquid chromatography (HPLC) $(11,12)$ and ultraviolet-visible spectrophotometry (UV-Vis) (13) are two commonly used methods for detecting Levofloxacin. Furthermore, several studies have determined the 
concentration and pharmacokinetics of Levofloxacin in serum or plasma $(14,15)$. However, few studies have been published that focused on a comparison of HPLC with UV-Vis, which is the preferred method for determination of Levofloxacin. Levofloxacin has been mixed with novel synthetic composite scaffolds that contained several components (10). However, where there is a lot of impurity interference in the samples, this has the tendency to increase the difficulty of detection (16). The aim of the present study was therefore to compare HPLC with $\mathrm{UV}-\mathrm{V}$ is for determining the level of Levofloxacin released from mesoporous silica microspheres/n-HA composite scaffolds in order to verify which of the two methods is preferable for this drug-delivery system.

\section{Materials and methods}

Equipment. Chromatographic analysis was performed with the use of a Shimadzu liquid chromatograph equipped with a model LC-2010AHT gradient pump, CBM-20A system controller and Shimadzu CLASS-VP UV-Visible detector (Shimadzu Corporation, Kyoto, Japan). The present study also utilized a Sigma D-37520 high-speed centrifuge (Merck KGaA, Darmstadt, Germany), a Kunshan Shu Mei KQ2200B ultrasonic cleaner (Kunshan Ultrasonic Instruments Co., Ltd., Kunshan, China), a Mettler-Toledo one-hundred-thousandth electronic balance (Mettler-Toledo $\mathrm{GmbH}$, Greifensee, Switzerland), an Elix10 water purification system (Milli-Q Gradient A10; EMD Millipore, Billerica, MA, USA), and a UV-2600 UV-Vis spectrophotometer (Shimadzu Corporation). Sample separation was performed on a Sepax BR-C18 column (250x4.6 mm; Sepax Technologies, Inc., Newark, DE, USA), with a particle diameter of $5 \mu \mathrm{m}$.

Chemicals. Levofloxacin was purchased from the National Institutes for Food and Drug Control (cat. no. 130455-201106; Beijing, China). Ciprofloxacin was purchased from Sigma-Aldrich; Merck KGaA (cat no. 17850-5G-F) and applied as the internal standard. Methanol [high-performance liquid chromatography (HPLC)-grade] and tetrabutylammonium bromide (analytically pure) were purchased from Merck KGaA.

Chromatography. The chromatographic separations were performed using a Sepax BR-C18 column $(250 \times 4.6 \mathrm{~mm}$; Sepax Technologies, Inc.) with $5-\mu \mathrm{m}$ particle diameter. The column temperature was set at $40^{\circ} \mathrm{C}$, and the mobile phase was prepared by mixing $0.01 \mathrm{~mol} / 1 \mathrm{KH}_{2} \mathrm{PO}_{4}$, methanol and $0.5 \mathrm{~mol} / 1$ tetrabutylammonium hydrogen sulphate (proportions, 75:25:4). This was delivered at a flow rate of $1 \mathrm{ml} / \mathrm{min}$. The wavelength of the detector was set at $290 \mathrm{~nm}$, and the injection volume was either $20 \mu \mathrm{l}$ for associated substances, or $10 \mu \mathrm{l}$ for assay determination.

Synthesis of novel composite scaffolds. The specific production method was described in detail in our previous article (10). Briefly, MSNs were synthesized according to the protocol reported by Argyo et al (17). A total of $0.2 \mathrm{~g}$ cetyltrimethylammonium bromide (CTAB) was combined with $0.75 \mathrm{ml} 2 \mathrm{~mol} / \mathrm{l}$ sodium hydroxide $(\mathrm{NaOH})$, and the resultant solution was then added to $100 \mathrm{ml} \mathrm{H}_{2} \mathrm{O}$ with constant stirring. Subsequently,
$20 \mathrm{ml} \mathrm{n}$-hexane was added into the above solution with stirring $(400 \mathrm{rpm})$. Iron (II,III) oxide $\left(\mathrm{Fe}_{3} \mathrm{O}_{4}\right)$ was stabilized with oleic acid. Ethyl acetate $(2 \mathrm{ml})$ and $0.5 \mathrm{ml}$ tetraethyl orthosilicate were added to the solution at $70^{\circ} \mathrm{C}$ for $3 \mathrm{~h}$. CTAB and $\mathrm{Fe}_{3} \mathrm{O}_{4}$ separated out as nanocrystals when the $\mathrm{pH}$ value of the solution was decreased. The solid product was obtained by filtering, prior to drying under vacuum at room temperature, and these were named as MSNs. Finally, MSNs were resolved in suspension and Levofloxacin $(1,500 \mu \mathrm{g} / \mathrm{ml})$ was loaded by electrostatic attraction.

The n-HA/PU composite porous scaffolds were successfully synthesized using the in situ foaming method $(13,18)$. Initially, $30 \mathrm{~g}$ castor oil was combined with $40 \mathrm{~g} \mathrm{n}-\mathrm{HA}$ particles in a nitrogen atmosphere and thorough stirring. Subsequently, $30 \mathrm{~g}$ isophorone diisocyanate was added to the suspension at $70^{\circ} \mathrm{C}$ for $3 \mathrm{~h}$ in order to obtain the prepolymer, and $1 \mathrm{ml}$ 1,4-butanediol was used to extend the prepolymer. During this step, the n-HA/PU composite scaffolds were actually obtained. The n-HA/PU was cut into small cuboids (10x6x6 mm) and immersed into the Levofloxacin-MSN suspension at $25^{\circ} \mathrm{C}$ for $30 \mathrm{~min}$, prior to drying in a vacuum oven at $40^{\circ} \mathrm{C}$. Following completion of the above steps, $1 \mathrm{mg}$ Levofloxacin-MSN-n-HA/PU (Lev@ MSN/n-HA/PU) was successfully synthesized.

Preparation of the standard solution. Levofloxacin (30.00 mg) was weighed precisely and dissolved in simulated body fluid (SBF; Hangzhou Haoxin Biotech, Co., Ltd., Hangzhou, China). Subsequently, the Levofloxacin solution was transferred to a $10 \mathrm{ml}$ volumetric flask and used to obtain a standard solution (3 $\mathrm{mg} / \mathrm{ml}$ Levofloxacin). The standard solution was diluted into 14 different concentration gradients: 300, 200, 100, 50, 25, $10,5,2.5,1,0.5,0.25,0.1,0.05$ and $0.01 \mu \mathrm{g} / \mathrm{ml}$. Ciprofloxacin (25.00 mg) was also weighed accurately and dissolved in methanol. The concentration of ciprofloxacin was adjusted to $500 \mu \mathrm{g} / \mathrm{ml}$, and this was used as the internal standard.

Wavelength selection of $U V$-Vis. The standard solution of Levofloxacin was prepared as described above. High (50 $\mu \mathrm{g} / \mathrm{ml})$, medium $(25 \mu \mathrm{g} / \mathrm{ml})$, and low $(5 \mu \mathrm{g} / \mathrm{ml})$ concentrations were selected. After the instrument was calibrated to zero, the maximum absorption wavelength was determined by scanning the standard levofloxacin solutions at 200-400 nm.

Establishment of the standard curve by HPLC. A total of 14 different concentrations of the Levofloxacin standard solutions (10 $\mu \mathrm{l}$ ) were respectively added into $100 \mu \mathrm{l}$ blank SBF at room temperature. Subsequently, $10 \mu \mathrm{l}$ ciprofloxacin was added into the working solution as an internal standard. The working solution was vortex-mixed for $5 \mathrm{~min}$, and $800 \mu \mathrm{l}$ dichloromethane was subsequently added. The solution was further vortex-mixed for $5 \mathrm{~min}$, and centrifuged at 7,155 $\mathrm{x}$ g for $5 \mathrm{~min}$ at $25^{\circ} \mathrm{C}$. An aliquot $(750 \mu \mathrm{l})$ of supernatant was extracted, and dried with nitrogen in a $50^{\circ} \mathrm{C}$ water bath. A total of $100 \mu \mathrm{l}$ of the mobile phase was added into the tube for redissolution. The supernatant was mixed in an ultrasonicator, vortex-mixed and centrifuged at $16,099 \mathrm{x}$ g for $10 \mathrm{~min}$ at $25^{\circ} \mathrm{C}$. An aliquot $(10 \mu \mathrm{l})$ of the supernatant was injected into a Sepax BR-C18 column (250x4.6 mm; Sepax Technologies, Inc., Newark, DE, USA) with a constant flow rate of $1 \mathrm{ml} / \mathrm{min}$. Each concentration of 


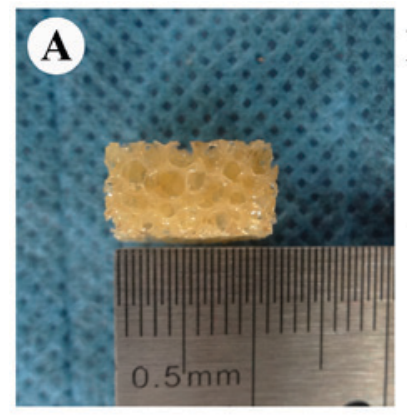<smiles></smiles>

Ciprofloxacin

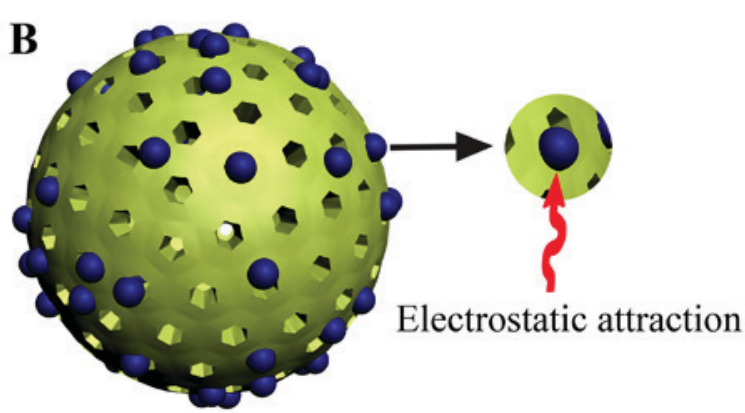

Levofloxacin

Figure 1. Structural and morphological characterization of the novel synthetic mesoporous silica microspheres. (A) Appearance of the n-HA/PU composite porous scaffolds. (B) Diagrammatic representation of the mesoporous silica microspheres. (C) Molecular structural formula of ciprofloxacin. (D) Molecular structural formula of Levofloxacin. n-HA/PU, nano-hydroxyapatite/polyurethane.

the standard product was analyzed 3 times. The ratio of the sum-of-peak areas of Levofloxacin to ciprofloxacin was set as the vertical axis $(y)$ in the standard curve, while the concentration of Levofloxacin was set as the horizontal axis $(x)$. Linear regression analysis was performed using the weighted least-square method $\left(\mathrm{W}=1 / \mathrm{C}^{2}\right)$.

Establishment of standard curve by $U V$-Vis. Blank SBF (3 ml) was scanned using UV-Vis, and the instrument was calibrated to zero. Different concentrations $(0.5,2.5,5,12.5$, 25 and $50 \mu \mathrm{g} / \mathrm{ml}$ ) of the standard product were scanned by UV-Vis. The absorbance value was set as the vertical axis $(y)$ in the standard curve, while the concentration of Levofloxacin was set as the horizontal axis $(x)$. Linear regression analysis was performed using the weighted least-square method $\left(\mathrm{W}=1 / \mathrm{C}^{2}\right)$.

Recovery of the two methods. Three different concentration levels of Levofloxacin (low, $5 \mu \mathrm{g} / \mathrm{ml}$; middle, $25 \mu \mathrm{g} / \mathrm{ml}$; high, $50 \mu \mathrm{g} / \mathrm{ml}$ ) were prepared, respectively. As quality control samples, these were processed and tested using HPLC in accordance with the method described above. The samples were also investigated by UV-Vis. The ratio between the measured concentration and the theoretical concentration was regarded as the method recovery rate.

Real sample determination. All the 1 mg Lev@ MSNs/ $\mathrm{n}-\mathrm{HA} / \mathrm{PU}$ composite porous scaffolds $(\mathrm{n}=10)$ were sterilized with $\gamma$-cobalt 60 radiation. The scaffolds were subsequently immersed in EP tubes containing $3 \mathrm{ml}$ modified SBF and incubated for $24 \mathrm{~h}$ at $37^{\circ}$. The scaffolds were subsequently removed. The leach liquor was placed in a refrigerator at $-20^{\circ} \mathrm{C}$ prior to testing. Each sample was determined 3 times. Sample processing for the UV-Vis method was performed as follows: The leach liquor was diluted 100 times with SBF, and $3 \mathrm{ml}$ of the resultant solution was placed in a quartz cell, and scanned at a specific wavelength. The absorbance values were then recorded, and a standard curve was then used in order to calculate the antibiotic concentrations.

Statistical analysis. SPSS version 19.0 statistical software (IBM Corp., Armonk, NY, USA) was used for statistical analysis. All quantitative data are presented as the mean \pm standard deviation. Linear regression was used to establish the standard curve of the HPLC and UV-Vis methods, respectively. Paired Student's t-test was used to compare the mean concentration obtained from the HPLC and UV-Vis methods. Pearson's correlation analysis was used to analyze the concentration detected by HPLC and UV-Vis methods. The relative standard deviation (RSD) was calculated according to the following equation: Standard deviation/calculated arithmetic mean $\mathrm{x} 100 \%$. $\mathrm{P}<0.05$ was considered to indicate a statistically significant value.

\section{Results}

Structural characterization of novel composite scaffolds. The novel biodegradable composite scaffolds containing a large quantity of MSNs were successfully constructed, and the n-HA/PU was cut into small cuboids (size, 10x6x6 mm). A large number of pores were observed in the material. The appearance of the n-HA/PU composite porous scaffolds is presented in Fig. 1A, and a three-dimensional structural diagram of newly fabricated mesoporous silica microspheres is presented in Fig. 1B. Numerous perforated channels were observed above the mesoporous silica microspheres. A large quantity of Levofloxacin was attached to the surface of the microspheres through electrostatic attraction. Ciprofloxacin was set as the internal standard in the process of determination; the molecular structural formula of Ciprofloxacin is presented in Fig. 1C. Likewise, the molecular structural formula of Levofloxacin is presented in Fig. 1D. 
A

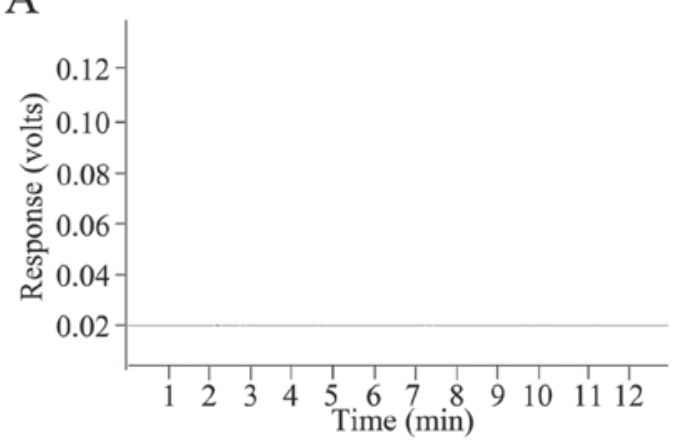

$\mathrm{C}$

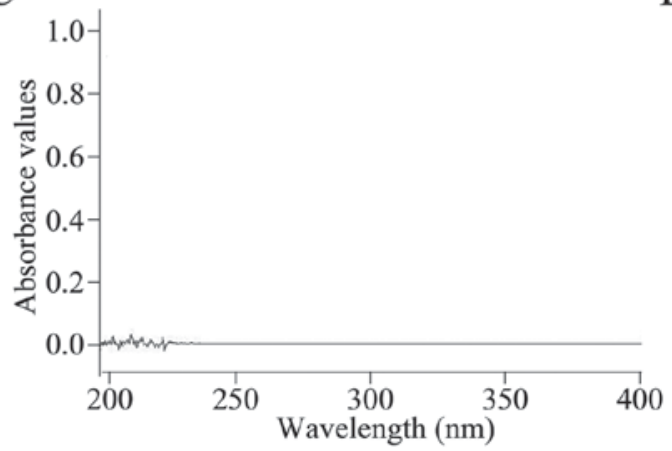

B

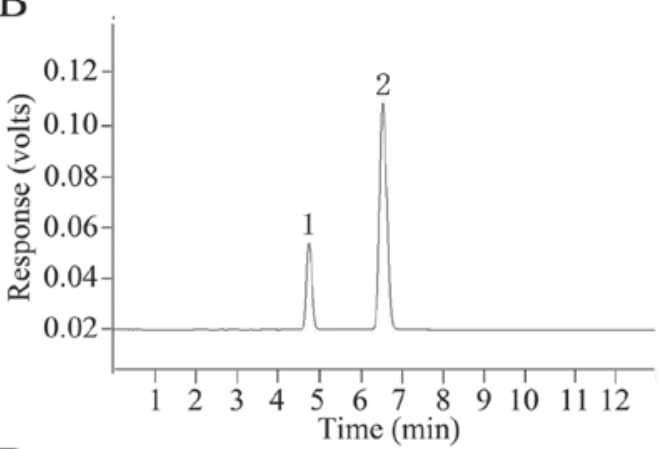

$\mathrm{D}$

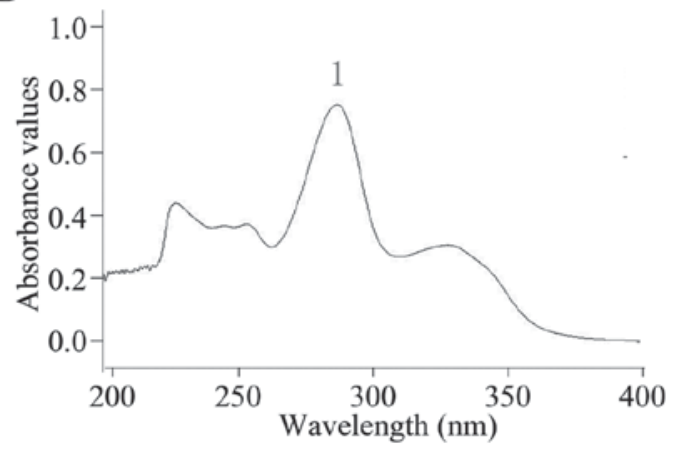

Figure 2. High-performance liquid chromatography chromatograms of each group. (A) Chromatogram of blank simulated body fluid. (B) Chromatograms of Levofloxacin (peak 1) and ciprofloxacin (peak 2). Also presented are the performances of (C) the blank control group and (D) the experimental group (peak 1 represents Levofloxacin).

Specificity of HPLC. The blank SBF containing no antibiotics was regarded as the blank control. Following its examination via HPLC, no clear indication of impurity interference could be identified on the chromatograms (Fig. 2A). The standard solution of Levofloxacin and ciprofloxacin was examined by HPLC, and the results demonstrated that the retention times of Levofloxacin and ciprofloxacin were $\sim 4.84$ and $6.74 \mathrm{~min}$, respectively (Fig. 2B). These results demonstrated that the adjusted mobile phase was optimized for the detection of these composite scaffolds, as it presented the shortest retention time, an improved shape to the peaks, and good resolution of Levofloxacin and ciprofloxacin.

Wavelength of $U V$-Vis. SBF was used as the blank control group, and this was established as the experimental group following addition of the standard solution of Levofloxacin. The performance of the blank control group and the experimental group are presented in Fig. 2C and D. Subsequently, the samples were detected by UV-Vis. It was revealed that Levofloxacin at different concentrations had a maximum absorption wavelength of $288.4 \mathrm{~nm}$. Therefore, $288.4 \mathrm{~nm}$ was selected as the wavelength for $\mathrm{UV}-\mathrm{V}$ is detection of the samples.

Linearity of the standard curve. The ratio of the sum-of-peak areas of each of Levofloxacin and ciprofloxacin was set as the vertical axis (y) in the standard curve according to the HPLC method. The concentration of Levofloxacin was used as the horizontal axis $(x)$. Linear regression was calculated using the weighted least square method $\left(\mathrm{W}=1 / \mathrm{C}^{2}\right)$. The linear concentration range was identified to be $0.05-300 \mu \mathrm{g} / \mathrm{ml}$ for Levofloxacin. The regression equation of HPLC yielded values of $y=0.033 x+0.010$, with $R^{2}=0.9991$. The chromatograms of Levofloxacin at different concentrations, and ciprofloxacin are presented in Fig. 3A. The standard curve derived from the HPLC method is presented in Fig. 3B. The absorbance value recorded by UV-Vis was set as the vertical axis $(y)$ in the standard curve according to the UV-Vis method. The concentration of Levofloxacin was set along the horizontal axis $(x)$. The regression equation for the UV-Vis method was identified to be $y=0.065 x+0.017$, with $R^{2}=0.9999$. The linear concentration range was identified to be $2.5-50 \mu \mathrm{g} / \mathrm{ml}$ for Levofloxacin. The wavelength of Levofloxacin with different concentrations is presented in Fig. 3C, and the representative calibration curve for $\mathrm{UV}-\mathrm{V}$ is is presented in Fig. 3D.

Recovery of the two methods. The low, medium, and high $(5,25$ and $50 \mu \mathrm{g} / \mathrm{ml})$ concentrations of the three standard solutions were determined by HPLC. The recovery rates were $96.37 \pm 0.50,110.96 \pm 0.23$ and $104.79 \pm 0.06 \%$, respectively. The RSD values were revealed to be $0.52,0.21$ and $0.08 \%$, respectively. The same three concentrations of low, medium, and high $(5,25$ and $50 \mu \mathrm{g} / \mathrm{ml})$ standard solutions were also checked by UV-Vis. The recovery rates were demonstrated to be $96.00 \pm 2.00,99.50 \pm 0.00$, and $98.67 \pm 0.06 \%$, respectively. The associated RSD values were 2.08, 0.00 and $0.06 \%$, respectively. All the results of the recovery analysis met the requirement of the Chinese pharmacopoeia, i.e. 80-120\%. The measured concentrations and recovery of the two methods are presented in Table I.

Comparison of HPLC and UV-Vis. The mean concentration of 10 samples measured according to the UV-Vis method 
A

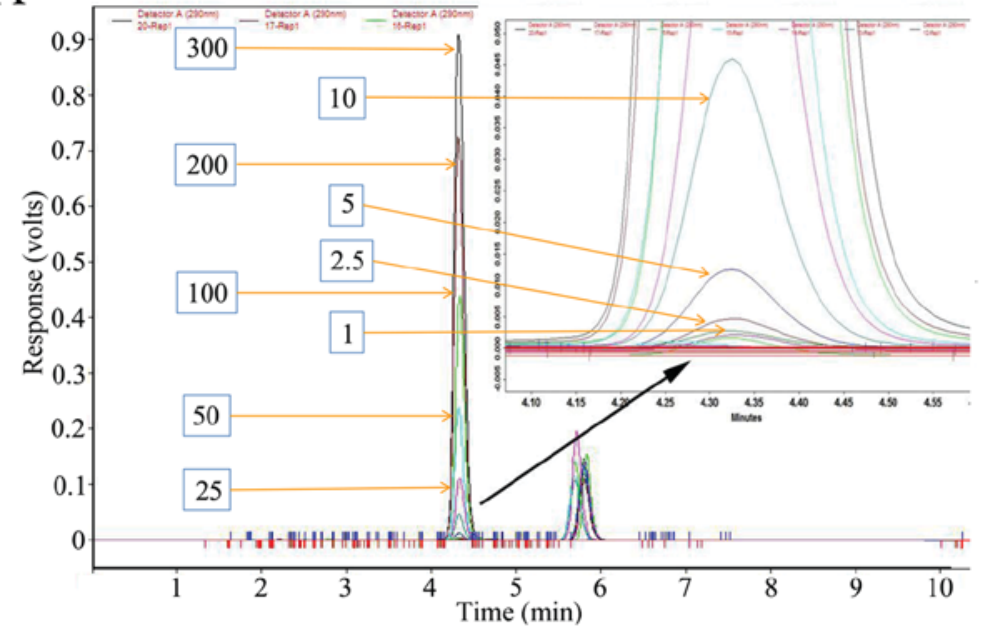

$\mathrm{C}$

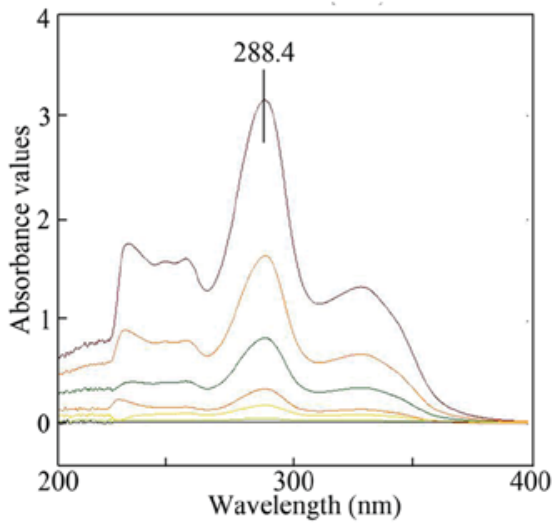

$\mathrm{B}$

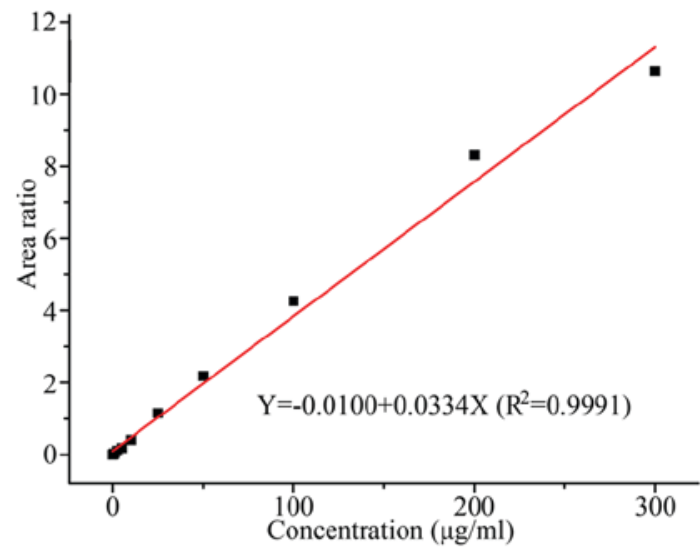

$\mathrm{D}$

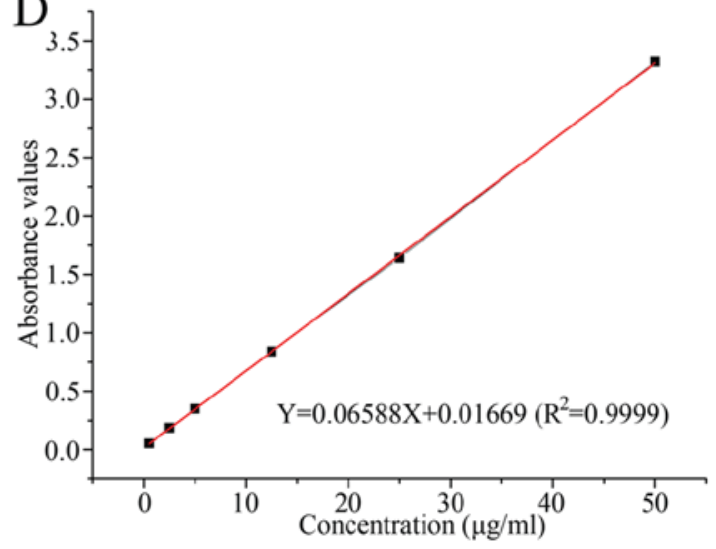

Figure 3. Establishment of the standard curves according to the HPLC and UV-Vis methods. (A) Chromatograms of Levofloxacin at different concentrations. (B) Standard curve generated by the HPLC method. (C) Wavelength spectra of Levofloxacin at the different concentrations investigated. (D) Standard curve generated by the UV-Vis method. HPLC, high-performance liquid chromatography; UV-Vis, ultraviolet-visible spectrophotometry.

was $187.93 \pm 33.52 \mu \mathrm{g} / \mathrm{ml}$, whereas the mean concentration of 10 samples measured using the HPLC method was $30.43 \pm 10.27 \mu \mathrm{g} / \mathrm{ml}$. Paired t-tests were used to compare the results of the two methods. A statistically significant difference was identified between the two methods $(t=20.17, \mathrm{P}<0.001$; Fig. 4A). The concentration of Levofloxacin measured by UV-Vis was set as the vertical axis (y), while the concentration of Levofloxacin measured by HPLC was set as the vertical axis $(x)$. These two results were used for correlation analysis. The linear equation was determined to be $y=2.94 x+98.57$, and the correlation coefficient (r) was identified to be 0.90 $(\mathrm{P}<0.001$; Fig. 4B). Characteristic parameters according to Lambert-Beer's law were examined. The absorbance value (A) has a logarithmic function with the reciprocal of transmittance (1/T) number (Fig. 4C). The peak wave of Levofloxacin could not be seen when the original sample was detected (Fig. 4D), however, after the sample was diluted the peak wave of Levofloxacin was observed (Fig. 4E).

\section{Discussion}

In our previous study, scaffolds based on novel composites were successfully synthesized, consisting of nano-HA, PU and MSNs. Levofloxacin was encapsulated in MSNs via electrostatic attraction (10). That publication demonstrated that these novel composite scaffolds presented an improved ability for inhibition of the inflammation reaction, and for treating chronic osteomyelitis with bone defects, compared with other groups. These novel composite scaffolds may have applicability in terms of their use as a local antibiotic delivery system for the treatment of chronic osteomyelitis and bone regeneration.

For the treatment of infectious diseases, the concentration of local drugs is required to reach the effective antibacterial concentration. In order to evaluate sustained release characteristics of these novel composite scaffolds as a local antibiotic delivery system in vitro and in vivo, it is necessary to optimize the detection method for accurate determination of the Levofloxacin concentration in local infectious soft tissue. Levofloxacin belongs to the third generation of fluoroquinolone antibiotics, demonstrating good antibacterial ability against Gram-positive and Gram-negative bacteria (19). The molecular mass of Levofloxacin is low; this therefore enables the compound to combine with MSNs through electrostatic attraction (20).

When the standard solutions of Levofloxacin were assessed, the two methods of HPLC and UV-Vis presented higher recovery rates and met the requirements of the Chinese pharmacopoeia. However, when the sample of novel composite scaffolds was measured by UV-Vis, the spectrum changed 
Table I. Measured concentration and recovery using the two methods.

\begin{tabular}{|c|c|c|c|c|c|}
\hline Method & $\begin{array}{l}\text { Real concentration } \\
\qquad(\mu \mathrm{g} / \mathrm{ml})\end{array}$ & $\begin{array}{l}\text { Measured concentration } \\
\qquad(\mu \mathrm{g} / \mathrm{ml})\end{array}$ & $\begin{array}{l}\text { Recovery } \\
(\%)\end{array}$ & $\begin{array}{c}\text { Mean recovery } \\
(\%)\end{array}$ & $\begin{array}{c}\mathrm{RSD} \\
(\%)\end{array}$ \\
\hline \multirow[t]{9}{*}{ HPLC } & 5 & 4.81 & 96.3 & $96.37 \pm 0.50$ & 0.52 \\
\hline & & 4.84 & 96.9 & & \\
\hline & & 4.79 & 95.9 & & \\
\hline & 25 & 27.67 & 110.69 & $110.96 \pm 0.23$ & 0.21 \\
\hline & & 27.76 & 111.09 & & \\
\hline & & 27.77 & 111.1 & & \\
\hline & 50 & 52.35 & 104.7 & $104.79 \pm 0.06$ & 0.08 \\
\hline & & 52.42 & 104.8 & & \\
\hline & & 52.41 & 104.8 & & \\
\hline \multirow[t]{9}{*}{ UV-Vis } & 5 & 4.83 & 96.0 & $96.00 \pm 2.00$ & 2.08 \\
\hline & & 4.72 & 94.0 & & \\
\hline & & 4.90 & 98.0 & & \\
\hline & 25 & 24.87 & 99.5 & $99.50 \pm 0.00$ & 0.00 \\
\hline & & 24.87 & 99.5 & & \\
\hline & & 24.87 & 99.5 & & \\
\hline & 50 & 49.34 & 98.7 & $98.67 \pm 0.06$ & 0.06 \\
\hline & & 49.34 & 98.7 & & \\
\hline & & 49.31 & 98.6 & & \\
\hline
\end{tabular}

RSD, relative standard deviation; HPLC, high-performance liquid chromatography; UV-Vis, ultraviolet-visible spectrophotometry.

significantly compared with the standard products. The mean concentration $(30.43 \pm 10.27 \mu \mathrm{g} / \mathrm{ml})$ of the samples determined by HPLC was significantly lower compared with the mean concentrations $(187.93 \pm 33.52 \mu \mathrm{g} / \mathrm{ml})$ of the samples determined by UV-Vis. It is considered that, as far as impurity interference absorbance of Levofloxacin is concerned, impurities can interfere with the accuracy of the UV-Vis measurements. The working principle of UV-Vis follows the Lambert-Beer law (21). The absorbance value (A) has a logarithmic function with the reciprocal of transmittance $(1 / \mathrm{T})$ number, so the absorbance value and concentration are linear only within a relatively small range: $0.3-0.7$ (22). This principle may explain why the UV-Vis method has a narrower linear range than HPLC. The range of linearity for UV-Vis was identified to be $2.5-50 \mu \mathrm{g} / \mathrm{ml}$ for Levofloxacin. The range of linearity for HPLC was identified to be $0.05-300 \mu \mathrm{g} / \mathrm{ml}$ for Levofloxacin. If the concentration of samples was too high $(>50 \mu \mathrm{g} / \mathrm{ml}$ in the present study), it is imprecise for measuring the concentration of levofloxacin by UV-Vis. Furthermore, it is considered that the multiple impurities of samples interference absorbance of Levofloxacin. The multiple impurities increase the absorbance value. The recovery rate of the UV-Vis method was determined to be between 96 and $98 \%$. When determining the recovery rate of the two methods, three different concentration levels of standard Levofloxacin were prepared respectively. These samples didn't contain impunities. However, when determining the concentration of levofloxacin released from the composite scaffolds, the composite scaffolds were initially immersed in SBF, the samples contained the multiple impurities which interference absorbance of Levofloxacin. Although $\mathrm{UV}-\mathrm{V}$ is revealed a satisfactory recovery rate, the concentration of Levofloxacin calculated was therefore not accurate. The concentration of the identical sample detected by the UV-Vis method was significantly higher compared with that of the HPLC method. The results of the two methods exhibited a positive correlation. Impurities in the sample may increase the absorbance of UV-Vis and the linear range of UV-Vis is narrow. With a difference in drug loading, a large difference ensues in terms of the amount of drug-sustained release, and this would fall outside the linear range of UV-Vis. These factors thereby result in an inability of the UV-Vis method to accurately measure the drug concentration.

The wavelength of Levofloxacin reached a maximum at $288.4 \mathrm{~nm}$. First, the undiluted solution was assessed directly by UV-Vis, and due to impurity interference and the high drug concentration employed, which exceeded the response absorbance of the UV-Vis method, the wavelength of Levofloxacin disappeared. The wavelength of Levofloxacin reappeared following dilution of the sample. Although the absorbance value lay within the linear range, the impurity resulted in an increase in the absorbance value, and the finally calculated concentration of Levofloxacin was higher than the real concentration. Therefore, the UV-Vis method could not meet all the requirements for the measurement.

In the preliminary experiments for the present study, the mobile phase of the HPLC method comprised a $0.01 \mathrm{~mol} / 1$ potassium dihydrogen phosphate-methanol solution. The HPLC chromatograms revealed that the chromatographic peak of Levofloxacin was not completely separated from the chromatographic peak associated with impurity, and furthermore, the chromatographic peak was irregular. The chromatographic performance was significantly improved following the addition 
A

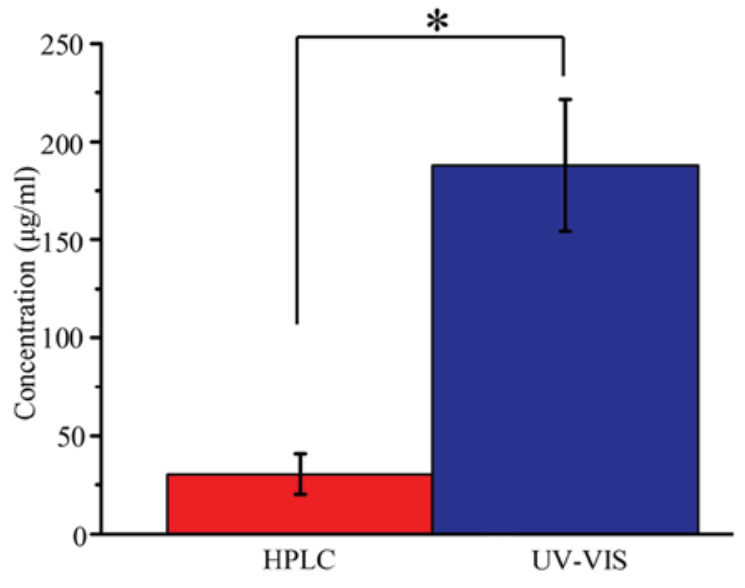

C

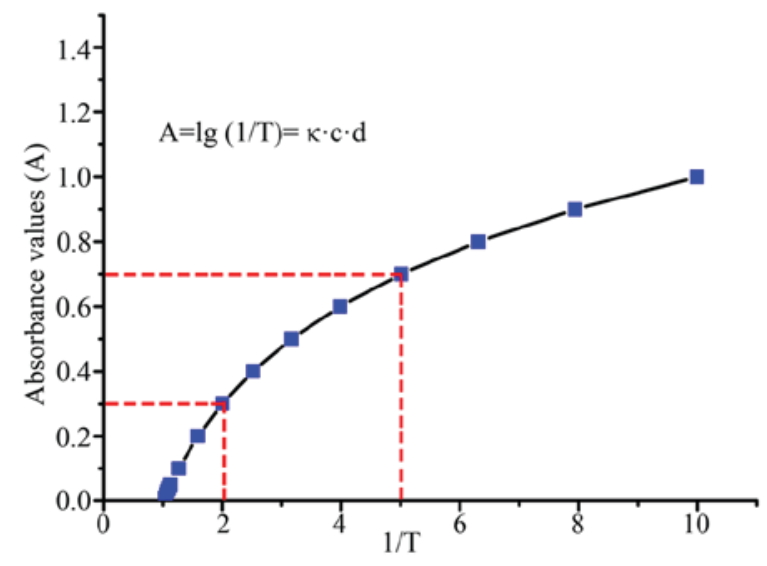

B

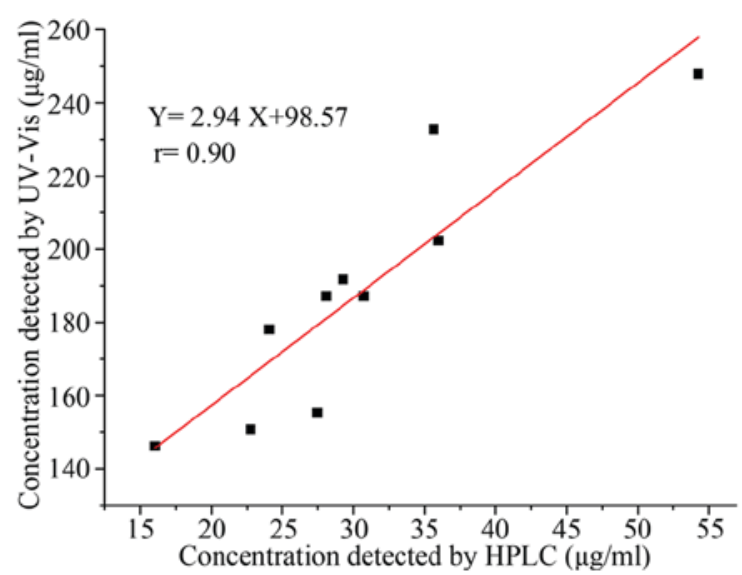

D

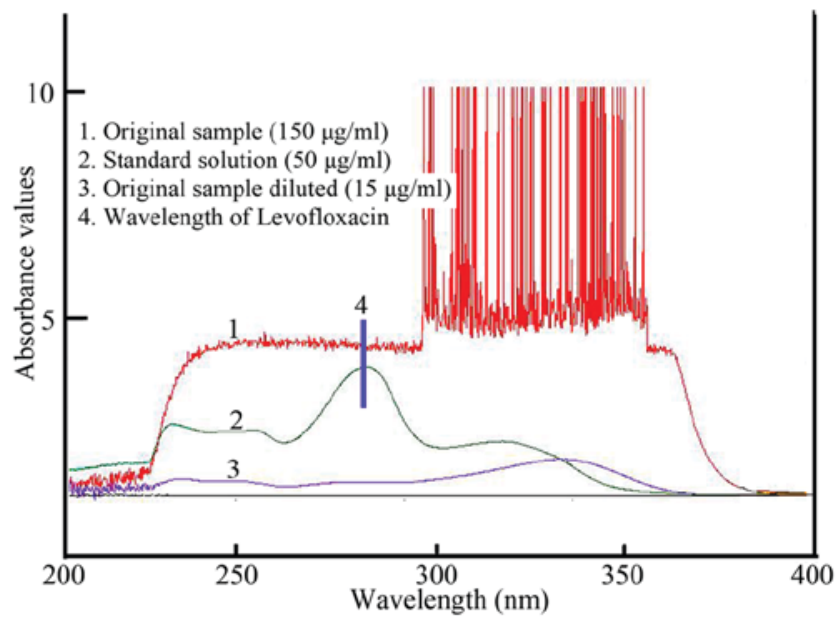

$\mathbf{E}$

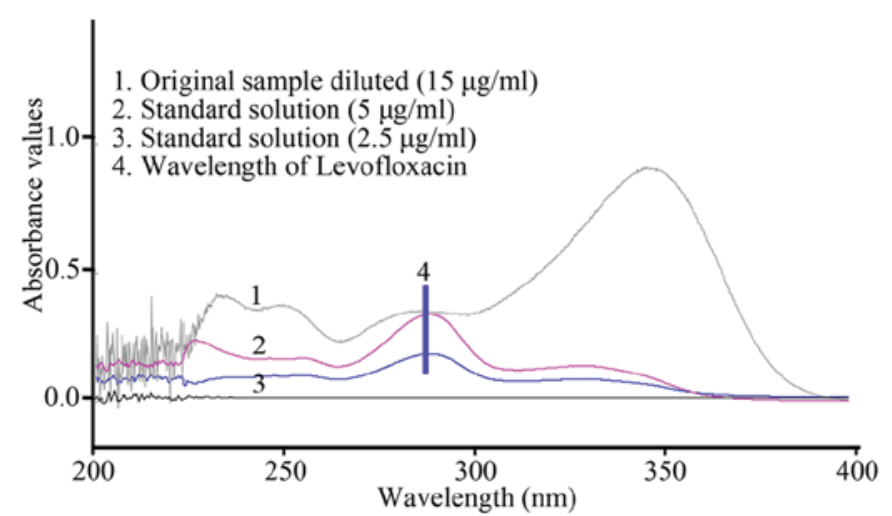

Figure 4. Comparison of HPLC and UV-Vis. (A) The mean concentration of 10 samples for the HPLC and UV-Vis methods. (B) Correlation analysis of both methods. (C) Characteristic parameters according to Lambert-Beer's law are presented. (D) Results of samples containing different concentration of Levofloxacin were determined by UV-Vis (trace 1, the original sample; trace 2, $50 \mu \mathrm{g} / \mathrm{ml}$ standard solution; trace 3, the sample was diluted 100 times; trace 4, wavelength of Levofloxacin). (E) The wavelength of Levofloxacin appeared after the sample was diluted (trace 1, dilution of the sample 100 times; trace 2 , $5 \mu \mathrm{g} / \mathrm{ml}$ standard solution; trace $3,2.5 \mu \mathrm{g} / \mathrm{ml}$ of standard solution; '4' indicates the wavelength of Levofloxacin). HPLC, high-performance liquid chromatography; UV-Vis, ultraviolet-visible spectrophotometry.

of $0.5 \mathrm{~mol} / 1$ tetrabutylammonium bromide into the mobile phase $(23,24)$. Finally, the mobile phase consisting of $0.01 \mathrm{~mol} / \mathrm{l}$ potassium dihydrogen phosphate, methanol and $0.5 \mathrm{~mol} / \mathrm{l}$ tetrabutylammonium bromide $(75: 25: 4)$ met with the requirements, and the chromatographic peaks of Levofloxacin and ciprofloxacin were separated from impurities. The chemical properties of ciprofloxacin are similar to those of Levofloxacin, and so the former was regarded as the internal standard (25). The two compounds are highly soluble in organic solvents. Therefore, dichloromethane was chosen as the extractant. In order to ensure sufficient extraction of the antibiotic in the sample, the ratio of sample to extractant was set as 1:8 (26). The recoveries of the HPLC method ranged from 96-105\%, reaching the requirements of the Chinese pharmacopoeia, i.e. 80-120\%. 
HPLC and UV-Vis are two commonly used methods for detecting Levofloxacin. The advantage of the UV-Vis method is that it is simple to operate, and is relatively inexpensive. A number of previous studies used UV-Vis to investigate the sustained release properties of drugs loaded on the composite composites $(27,28)$. However, a number of disadvantages also exist: Impurities in the sample could not be ruled out, and these may increase the calculated concentration; furthermore, the linear range is narrow. Therefore, it is not clear whether the studies using UV-Vis to measure drug concentrations are reliable. An aim of the present study was to investigate which method is preferable for this drug delivery system. Another purpose of the present study was to explain why the concentration of Levofloxacin detected by these two methods was different.

In conclusion, in the present study, the concentration of Levofloxacin was measured by HPLC and UV-Vis. The aim of the present study was to compare the techniques of HPLC and UV-Vis. The findings demonstrated that although $\mathrm{UV}-\mathrm{V}$ is is simple to operate, and the expenses are low, it is not accurate to measure the concentration of drugs loaded on the biodegradable composite composites. The present study provides guidance on which methods should be selected for investigating the sustained release properties of drugs in tissue engineering. The HPLC method could be used to separate Levofloxacin from various impurities in the chromatographic column, which eliminated the interference of impurities with Levofloxacin. As a method, HPLC exhibited the advantages of high precision and high recovery. Therefore, HPLC is the preferred method to evaluate sustained release characteristics of Levofloxacin released from mesoporous silica microspheres/ n-HA composite scaffolds.

\section{Acknowledgements}

Not applicable.

\section{Funding}

The current study was supported by grants from the Key Research and Development Program of Shandong Province (grant no. 2017GSF218065) and Natural Science Foundation of Shandong Province (grant no. ZR201702140118).

\section{Availability of data and materials}

The datasets used and/or analyzed during the current study are available from the corresponding author on reasonable request.

\section{Authors' contributions}

QW and XZ designed the experiments. QW, GW and SX performed the experiments. YZ analyzed and interpreted the data. QW prepared the manuscript. All authors read and approved the final manuscript.

\section{Ethics approval and consent to participate}

Not applicable.

\section{Patient consent for publication}

Not applicable.

\section{Competing interests}

The authors declare that they have no competing interests.

\section{References}

1. Das A, Mukherjee J, Dey G, Sarkar AK, Sahoo BK Chakrabarty US, Nandi U and Pal TK: Bioequivalence study of levofloxacin tablets in healthy Indian volunteers using HPLC. Arzneimittelforschung 61: 61-65, 2011.

2. Wimer SM, Schoonover L and Garrison MW: Levofloxacin: A therapeutic review. Clin Ther 20: 1049-1070, 1998.

3. Carral N, Lukas JC, Oteo I and Suarez E: Impact of poor compliance with levofloxacin and moxifloxacin on respiratory tract infection antimicrobial efficacy: A pharmacokinetic/pharmacodynamic simulation study. Int J Antimicrob Agents 45: 79-83, 2015.

4. Sun H, Wang H and Ge X: Simultaneous determination of the combined drugs of ceftriaxone sodium, metronidazole, and levofloxacin in human urine by high-performance liquid chromatography. J Clin Lab Anal 26: 486-492, 2012.

5. Wang SS, Ratliff PD and Judd WR: Retrospective review of ceftriaxone versus levofloxacin for treatment of E. coli urinary tract infections. Int J Clin Pharm 40: 143-149, 2018.

6. Cicuéndsez M, Izquierdo-Barba I, Portolés MT and Vallet-Regí M: Biocompatibility and levofloxacin delivery of mesoporous materials. Eur J Pharm Biopharm 84: 115-124, 2013.

7. Croissant JG, Fatieiev Y, Almalik A and Khashab NM: Mesoporous silica and organosilica nanoparticles: Physical chemistry, biosafety, delivery strategies, and biomedical applications. Adv Healthc Mater: 7, 2018 doi: 10.1002/adhm.201700831.

8. Shi S, Chen F and Cai W: Biomedical applications of functionalized hollow mesoporous silica nanoparticles: Focusing on molecular imaging. Nanomedicine (Lond) 8: 2027-2039, 2013.

9. Guisasola E, Asín L, Beola L, de la Fuente JM, Baeza A and Vallet-Regí M: Beyond traditional hyperthermia: In vivo cancer treatment with magnetic-responsive mesoporous silica nanocarriers. ACS App Mater Interfaces 10: 12518-12525, 2018.

10. Wang Q, Chen C, Liu W, He X, Zhou N, Zhang D, Gu H, Li J, Jiang $\mathrm{J}$ and Huang W: Levofloxacin loaded mesoporous silica microspheres/nano-hydroxyapatite/polyurethane composite scaffold for the treatment of chronic osteomyelitis with bone defects. Sci Rep 7: 41808, 2017.

11. Szerkus O, Jacyna J, Gibas A, Sieczkowski M, Siluk D, Matuszewski M, Kaliszan R and Markuszewski MJ: Robust HPLC-MS/MS method for levofloxacin and ciprofloxacin determination in human prostate tissue. J Pharm Biomed Anal 132: 173-183, 2017.

12. Gao XX, Yao GC, Guo N, An F and Guo XJ: A simple and rapid high performance liquid chromatography method to determine levofloxacin in human plasma and its use in a bioequivalence study. Drug Discov Ther 1: 136-140, 2007.

13. Netz DJ, Sepulveda P, Pandolfelli VC, Spadaro AC, Alencastre JB, Bentley MV and Marchetti JM: Potential use of gelcasting hydroxyapatite porous ceramic as an implantable drug delivery system. Int J Pharm 213: 117-125, 2001.

14. Siewert S: Validation of a levofloxacin HPLC assay in plasma and dialysate for pharmacokinetic studies. J Pharm Biomed Anal 41: 1360-1362, 2006.

15. Arayne MS, Sultana N and Siddiqui FA: Optimization of levofloxacin analysis by RP-HPLC using multivariate calibration technique. Pak J Pharm Sci 20: 100-106, 2007.

16. Lalitha Devi $M$ and Chandrasekhar KB: A validated stability-indicating RP-HPLC method for levofloxacin in the presence of degradation products, its process related impurities and identification of oxidative degradant. J Pharm Biomed Anal 50: 710-717, 2009.

17. Argyo C, Weiss V, Bräuchle C and Bein T: Multifunctional mesoporous silica nanoparticles as a universal platform for drug delivery. Chemistry Materials 26: 435-451, 2014.

18. Sepulveda P, Binner JG, Rogero SO, Higa OZ and Bressiani JC: Production of porous hydroxyapatite by the gel-casting of foams and cytotoxic evaluation. J Biomed Mater Res 50: 27-34, 2000. 
19. GhaffarKA,Hussein WM, KhalilZG, Capon RJ,Skwarczynski M and Toth I: Levofloxacin and indolicidin for combination antimicrobial therapy. Curr Drug Deliv 12: 108-114, 2015.

20. Guenter SG, Iven H, Boos C, Bruch HP and Muhl E: Pharmacokinetics of levofloxacin during continuous venovenous hemodiafiltration and continuous venovenous hemofiltration in critically ill patients. Pharmacotherapy 22: 175-183, 2002.

21. Langergraber G, Fleischmann N and Hofstädter F: A multivariate calibration procedure for UV/VIS spectrometric quantification of organic matter and nitrate in wastewater. Water Sci Technol 47: 63-71, 2003.

22. Nagaraja P, Al-Tayar NG, Shivakumar A, Shrestha AK and Gowda AK: A simple and sensitive spectrophotometric method for the determination of trace amounts of nitrite in environmental and biological samples using 4-amino-5-hydroxynaphthalene-2,7-disulphonic acid monosodium salt. Spectrochim Acta A Mol Biomol Spectrosc 75: 1411-1416, 2010.

23. Chamseddin $\mathrm{C}$ and Jira TH: Comparison of the chromatographic behavior of levofloxacin, ciprofloxacin and moxifloxacin on various HPLC phases. Pharmazie 66: 244-248, 2011.

24. Nguyen HA, Grellet J, Ba BB, Quentin C and Saux MC: Simultaneous determination of levofloxacin, gatifloxacin and moxifloxacin in serum by liquid chromatography with column switching. J Chromatogr B Analyt Technol Biomed Life Sci 810: 77-83, 2004.
25. Watabe S, Yokoyama Y, Nakazawa K, Shinozaki K, Hiraoka R, Takeshita K and Suzuki Y: Simultaneous measurement of pazufloxacin, ciprofloxacin, and levofloxacin in human serum by high-performance liquid chromatography with fluorescence detection. J Chromatogr B Analyt Technol Biomed Life Sci 878: 1555-1561, 2010.

26. Ji HY, Jeong DW, Kim YH, Kim HH, Sohn DR and Lee HS: Hydrophilic interaction liquid chromatography-tandem mass spectrometry for the determination of levofloxacin in human plasma. J Pharm Biomed Anal 41: 622-627, 2006.

27. Borcan LC, Dudas Z, Len A, Fuzi J, Borcan F and Tomescu MC: Synthesis and characterization of a polyurethane carrier used for a prolonged transmembrane transfer of a chili pepper extract. Int J Nanomedicine 6: 7155-7166, 2018.

28. Rahimi S, Khoee S and Ghandi M: Development of photo and $\mathrm{pH}$ dual crosslinked coumarin-containing chitosan nanoparticles for controlled drug release. Carbohydr Polym 201: 236-245, 2018. Attribution 4.0 International (CC BY-NC 4.0) License 